\title{
ANÁLISE NUMÉRICA DOS ELETROFOREGRAMAS DE GLIADINAS DE CULTIVARES DE TRITICALE ${ }^{1}$
}

\author{
ELISEU BINNECK², JORGE LUIZ NEDEL ${ }^{3}$, ODIR A. DELLAGOSTIN, SILMAR T. PESKE ${ }^{3}$ E A.C.S.A. BARROS ${ }^{5}$
}

\begin{abstract}
RESUMO - Buscando automatizar o processo de identificação de cultivares pelo método de eletroforese de proteínas, o presente trabalho teve por objetivo avaliar a viabilidade da utilização de valores de intensidade das bandas como dados adicionais de polimorfismos na discriminação de cultivares e comparar o comportamento de vários coeficientes de similaridade na análise dos eletroforegramas de gliadinas. Os eletroforegramas de gliadinas de quatro cultivares de triticale estreitamente relacionadas foram comparados pela análise computacional utilizando seis coeficientes de similaridade binários (presença/ausência) (Jaccard, Sorensen-Dice, Nei \& Li, Simple Matching, Yule e Baroni-Urbani) e cinco quantitativos (Pearson product moment correlation, Spearman, Percent Similarity, Modified Morisita e Gower). As análises quantitativas dos eletroforegramas levaram em conta a intensidade das bandas, disposta em diferentes números de classes, possibilitando avaliar o efeito da variabilidade desse parâmetro. Foram utilizadas cerca de 60 amostras individuais de sementes de cada cultivar. Apesar do baixo polimorfismo intervarietal, os coeficientes médios de similaridade dentro das cultivares sempre foram maiores do que entre cultivares, o que indica o alto poder discriminatório dos testes. $\mathrm{O}$ estudo demonstra a viabilidade de identificar cultivares de triticale pela comparação numérica dos dados de eletroforese de gliadinas, obtidos das amostras, com uma biblioteca de eletroforegramas. Esse procedimento garante uma comparação mais objetiva dos eletroforegramas, em relação à análise visual. Foi constatado também, que a intensidade (densidade) das bandas nos eletroforegramas de gliadinas é muito variável, sendo portanto um parâmetro pouco confiável na análise de polimorfismos de gliadinas em triticale. Ainda com relação ao parâmetro intensidade, há uma perda progressiva na confiabilidade dos resultados de comparação com o aumento no número de classes de intensidade. Termos para indexação: coefícientes de similaridade, identificação de cultivares, eletroforese.
\end{abstract}

\section{NUMERICAL ANALYSIS OF GLIADINS ELETROPHOREGRAMS OF TRITICALE CULTIVARS}

\begin{abstract}
Aiming the automatization of cultivar identification by protein electrophoresis, the objective of this work was evaluate the feasibility of using band intensity values as polimorphism aditional data on cultivar discrimination and compare different similarity coefficients on the analysis of gliadins electophoregrams data. Electrophoregrams of gliadinas take from four cultivars of triticale closely related were compared by the computer-assisted analysis using six binary (presence/ absence) coefficients of similarity (Jaccard, Sorensen-Dice, Nei \& Li, Simple Matching, Yule and Baroni-Urbani) and five quantitative coefficients (Pearson product moment correlation, Spearman, Percent Similarity, Modified Morisita and Gower). The intensity of bands used on the quantitative analysis of electrophoregrams was distributed in different number of classes of intensity, making possible to evaluate the effect of variability on this parameter. It was used about 60 single seed samples of each cultivar. In spite of low inter-varietal polymorphism, the coefficient means within
\end{abstract}

\footnotetext{
Aceito para publicação em 06.03.2002; trabalho realizado com suporte financeiro da CAPES.

${ }^{2}$ Eng $^{\mathrm{O}} \mathrm{Agr}^{\mathrm{o}}$, Dr., Laboratório de Biotecnologia, Embrapa Soja, Cx. Postal 231,86001-970, Londrina-PR; e-mail: binneck@cnpso.embrapa.br

${ }^{3}$ Prof. Titular, PhD., Depto. de Fitotecnia, FAEM/UFPel, Cx. Postal 354,
}

Pelotas-RS; e-mail: jlnedel@ufpel.tche.br; peske@ufpel.tchel.br

${ }^{4}$ Prof., PhD. do Centro de Biotecnologia, Depto. de Botânica/UFPel; e-mail: odirad@terra.com.br

${ }_{5}^{5}$ Prof. Adjunto, Dr., Depto. de Fitotecnia, FAEM/UFPel; e-mail: acbarros@ufpel.tche.br 


\begin{abstract}
of the cultivars was always greater than between cultivars. That clearly shows the high discriminating power of the tests. With this study we demonstrate the viability of identifying triticale cultivars by the numeric comparison of gliadin electrophoresis data with a library of electrophoregrams. It allows a more objective comparison of the electrophoregrams, comparing to visual analysis. We also found a great variability on intensity (density) data of bands. The parameter intensity is therefore not much reliable on gliadins polymorphism analysis in triticale. Hence about the intensity, there is a progressive lost on reliability of the results as increase the number of intensity classes.

Index terms: similarity coefficients, cultivar identification, electrophoresis.
\end{abstract}

\section{INTRODUÇÃO}

A necessidade de métodos precisos e confiáveis para a identificação e avaliação da pureza genética de cultivares, cada vez mais numerosas, tem se tornado urgente com a introdução da lei de proteção de cultivares e conseqüente necessidade para a proteção dos genótipos. Além disso, como as cultivares são atualmente melhoradas para usos específicos, por exemplo, qualidade de panificação em trigo, qualidade de malte em cevada, é importante que a cultivar e a pureza da amostra possa ser apurada com exatidão no comércio.

A eletroforese de proteínas de reserva, como as gliadinas, que são encontradas em múltiplas formas moleculares na cariopse de triticale, constitui uma alternativa bastante atrativa para identificação de cultivares e estudos genéticos da espécie. Contudo, o eletroforegrama de gliadinas de cada cultivar consiste de 20 a 25 bandas que são freqüentemente muito próximas ou fusionadas, resultando num número bastante elevado quando várias cultivares são comparadas (algo em torno de 60 possíveis bandas). Com isso, a identificação correta de cada banda no eletroforegrama, a normalização frente a um padrão conhecido e a comparação com uma biblioteca de eletroforegramas conhecidos poder se tornar um trabalho tedioso, com resultados muitas vezes equivocados (Bietz \& Simpson, 1992)

Além disso, a observação de alguma variabilidade intravarietal nos eletroforegramas de proteínas (Singh et al., 1973) tem sido apontado como uma das restrições ao seu uso imediato na caracterização varietal pela União Internacional para Proteção das Novas Obtenção Vegetais (Union pour l'Obtentions dês Vegetales - UPOV (UPOV, 1988)). Em geral, no processo de melhoramento, as plantas são selecionadas e avaliadas para características morfológicas e fisiológicas semelhantes, conforme as características agronômicas e industriais desejadas, as quais muitas vezes são usadas como descritores na caracterização e/ou identificação da cultivar. No entanto, não há, necessariamente, correlação entre uni- formidade morfológica, fisiológica e padrão eletroforético de proteínas de reserva da semente, como é o caso das gliadinas (Cooke, 1984 e Cooke, 1995). Claramente, é necessário que a técnica utilizada para identificação varietal ou análise da pureza genética, leve em conta a existência dessa variabilidade. Ainda, as técnicas de eletroforese de proteínas têm apresentado algumas limitações em diferenciar algumas cultivares, pelo número limitado de polimorfismos encontrados.

Um outro fator preocupante é a dificuldade de normalização dos eletroforegramas obtidos em diferentes géis, principalmente quando se trata de géis confeccionados no próprio laboratório. Observando esses problemas, nessa investigação procurou-se avaliar a viabilidade em utilizar valores de intensidade das bandas como dados adicionais de polimorfismos na discriminação de cultivares e comparar o comportamento de vários coeficientes de similaridade na análise dos eletroforegramas de gliadinas, buscando automatizar o processo de identificação de cultivares por este método.

\section{MATERIAL E MÉTODOS}

Foram utilizadas as cultivares de triticale Embrapa 17, Embrapa 53, BR 4 e BRS 148, obtidas do Serviço de Produção de Sementes Básicas (SPSB), da Embrapa - Trigo, Passo Fundo. Sementes da classe genética dessas cultivares foram produzidas em 1998 em um ensaio regional em Pelotas, no campo experimental do Centro Agropecuário da Palma UFPel. Foram colhidas espigas de 30 plantas típicas de cada cultivar, sendo analisadas sementes de duas espigas por planta. Todas as sementes foram mantidas em câmara seca $\left(11^{\circ} \mathrm{C}\right.$ e $45 \%$ de UR) até a sua utilização. As análises do material biológico foram concluídas em até 18 meses após a colheita. A cultivar BRS 148 foi selecionada para ser utilizada como padrão de referência nos géis de eletroforese.

Cada semente inteira, com aproximadamente $40 \mathrm{mg}$, foi macerada entre duas folhas de papel germitest, com um alicate, e transferida para um tubo de microcentrífuga (eppendorf) 
de $1,5 \mathrm{ml}$. Nesse tubo foi adicionado $0,3 \mathrm{ml}$ da solução de extração (25\% de 2-cloroetanol e 0,05\% de metil green em água destilada). Após agitação vigorosa, dissolvendo completamente o conteúdo da semente na solução de extração, a mistura foi mantida por uma noite em temperatura ambiente $\left(20-22^{\circ} \mathrm{C}\right)$ com os tubos fechados. Posteriormente, o conteúdo foi submetido à centrifugação a 18.000 x g, sendo o sobrenadante utilizado para a eletroforese.

A mistura do gel consistiu de $10 \mathrm{~g}$ de acrilamida, $0,4 \mathrm{~g}$ de bisacrilamida, $6 \mathrm{~g}$ de uréia, $0,005 \mathrm{~g}$ de sulfato ferroso e $0,1 \mathrm{~g}$ de ácido ascórbico dissolvidos em um volume total de $100 \mathrm{ml}$ do tampão do gel (20ml de ácido acético glacial e $1 \mathrm{~g}$ de glicina em água destilada completando o volume de um litro). A solução resultante foi colocada em refrigerador para redução da temperatura até $4^{\circ} \mathrm{C}$ e, então, foram adicionados $100 \mathrm{ml} \mathrm{de}$ uma solução nova de persulfato de amônio a 10\% (mantida a $4^{\circ} \mathrm{C}$ ) e $300 \mathrm{ml}$ de TEMED, homogeneizando rapidamente, cuidando para não formar bolhas de ar. Os géis foram construídos com $11 \mathrm{~cm}$ de altura, $14 \mathrm{~cm}$ de largura e 1,5mm de espessura.

O tampão do eletrodo foi preparado com $4 \mathrm{ml}$ de ácido acético glacial e $0,4 \mathrm{~g}$ de glicina, diluídos em água destilada até completar o volume de um litro. A eletroforese foi conduzida a $250 \mathrm{~V}$ (voltagem constante), até a segunda banda (púrpura) do marcador methyl green chegar ao final do gel, com o eletrodo positivo na origem (topo) do gel. A eletroforese foi realizar no interior de uma câmara refrigerada para manter a temperatura do gel, durante a corrida, entre 15 e $20^{\circ} \mathrm{C}$.

Após a eletroforese, o gel foi removido das placas e colocado, por 24 horas, em bandeja de plástico contendo solução corante suficiente para cobri-lo. A solução corante foi preparada com a mistura de $10 \mathrm{ml}$ da solução CB $1 \%$ (1\% de Coomassie brilliant blue R250 em metanol) e $200 \mathrm{ml}$ da solução TCA 10\% (de ácido tricloroacético a 10\%). A bandeja foi mantida coberta, sendo submetida à agitação lenta por quatro horas e mantida em temperatura ambiente por mais 20 horas. Após este período, o gel foi lavado com água destilada para destacar as bandas.

As imagens foram capturadas e digitalizadas a partir dos géis frescos, colocados sobre uma plataforma de luz branca, utilizando o sistema Kodak EDAS 120 (Kodak Electrophoresis Documentation and Analysis System) e o programa Kodak 1D Image Analysis (Eastman Kodak Company, Rochester, USA), configurado para Coomassie Blue e exposição de 1/275 segundos. A imagens dos géis foram organizadas num sistema de base de dados e analisadas no programa Diversity Database ${ }^{\mathrm{TM}}$, versão 2.2 (Bio-Rad Laboratories). Após definidas as pistas (slots), efetuou-se a extração do background, baseado no perfil de cada pista, utilizando o método "Rolling Disk", sendo o raio do disco configurado para 20 pixels.

A normalização dos géis foi feita com base nos tipos de bandas. Para cada tipo de banda foi calculado o Rf, o qual foi designado ao mesmo tipo de banda nos demais géis. Os dados de posição e intensidade de cada banda foram obtidos a partir dos eletroforegramas de cada amostra de semente única, servindo para comparar a variabilidade existente dentro de cada cultivar e avaliar a proximidade genética entre as diferentes cultivares. Esses dados foram obtidos com o auxílio do Software Diversity Database ${ }^{\mathrm{TM}}$ e exportados para o Excel $^{\circ}$. A partir desses dados foram, calculadas as matrizes de similaridade, com o auxílio do programa MVSP $^{\odot}$ (MultiVariate Statistical Package; Kovach Computing Services, Wales, UK), que apresenta 23 opções de medidas de (dis)similaridade. Foram escolhidas seis medidas de similaridade binárias, ou seja, que consideram apenas o posicionamento e presença/ausência das bandas, e cinco medidas de similaridade que consideram a posição e intensidade das bandas. Os dendrogramas foram construídos no MVSP pelo método UPGMA (Unweighted Pair-Group Method using Aritmetic Average; média das distâncias) (Swofford \& Olsen, 1990 e Manly, 1994), mas devido ao número muito elevado de casos, não são mostrados neste trabalho. As médias e os desvios padrões das medidas de similaridades foram calculados com o auxílio do Excel $^{\circ}$.

Os coeficientes binários utilizados nesse trabalho foram: (i) coeficiente de Nei \& Li; (ii) coeficiente de Jaccard; (iii) coeficiente de Sorensen-Dice; (iv) coeficiente de concordância simples (Simple matching); (v) coeficiente de Yule; e (vi) coeficiente de Baroni-Urbani Buser. Os demais coeficientes, baseados na posição e intensidade das bandas, foram: (i) coeficiente de Pearson (Pearson product moment correlation coefficient); (ii) coeficiente de Spearman (Spearman rank order correlation coefficient); (iii) coeficiente de porcentagem de similaridade (Percent similarity coefficient); (iv) coeficiente de Morista modificado (Modified Morisita's coefficient); (v) coeficiente de Gower (Gower general similarity coefficient). Todos os coeficientes foram calculados pelos respectivos algoritmos dispostos no software MVSP $^{\odot}$ (Kovach, 1993).

Foram construídos gráficos para verificar a variação das intensidades das bandas ao longo dos eletroforegramas de cada cultivar, bem como para estabelecer a confiabilidade do parâmetro intensidade como uma medida de polimorfismo das gliadinas em triticale. Os gráficos foram construídos com o auxílio do software Statistica ${ }^{\circledR}$ (StatSoft, 1998). 


\section{RESULTADOS E DISCUSSÃO}

A análise dos eletroforegramas de gliadinas em gel de poliacrilamida geralmente requer a simplificação dos dados originados, pelo grande número de bandas polimórficas encontradas. Esses dados podem ser utilizados para calcular uma matriz de similaridade (Tabelas 1 e 2). Essa medidas de similaridade podem ser estabelecidas utilizando-se um grande número de coeficientes conhecidos. Podem ser utilizados coeficientes baseados apenas na presença ou ausência das

TABELA 1. Valores de similaridade comparando as amostras de semente única dentro da cultivar e entre cultivares de triticale, através de seis coeficientes binários (presença/ ausência).

\begin{tabular}{|c|c|c|c|c|}
\hline \multirow[b]{2}{*}{ Cultivares } & \multicolumn{4}{|c|}{ Similaridade (média \pm DP) } \\
\hline & $\begin{array}{c}\mathrm{BR} 4 \\
(n=54)\end{array}$ & $\begin{array}{l}\text { Emb } 17 \\
(n=56)\end{array}$ & $\begin{array}{l}\text { Emb } 53 \\
(n=57)\end{array}$ & $\begin{array}{l}\text { BRS } 148 \\
(n=58)\end{array}$ \\
\hline \multicolumn{5}{|c|}{ Coeficiente de Jaccard (Jaccard 1908). } \\
\hline Embrapa 17 & $0,70 \pm 0,07$ & \multicolumn{2}{|l|}{$0,89 \pm 0,08$} & \multirow[b]{3}{*}{$0,82 \pm 0,10$} \\
\hline Embrapa 53 & $0,65 \pm 0,06$ & $0,59 \pm 0,06$ & $0,94 \pm 0,06$ & \\
\hline BRS 148 & $0,60 \pm 0,07$ & $0,58 \pm 0,06$ & $0,50 \pm 0,06$ & \\
\hline \multicolumn{5}{|c|}{.... Nei \& Li's (Nei and Li 1979)... } \\
\hline BR 4 & \multicolumn{4}{|c|}{$0,94 \pm 0,05$} \\
\hline Embrapa 17 & $0,82 \pm 0,05$ & \multicolumn{2}{|l|}{$0,94 \pm 0,05$} & \multirow[b]{3}{*}{$0,90 \pm 0,06$} \\
\hline Embrapa 53 & $0,78 \pm 0,05$ & $0,74 \pm 0,05$ & $0,97 \pm 0,03$ & \\
\hline BRS & $0,75 \pm 0,05$ & $0,73 \pm 0,05$ & $0,66 \pm 0,06$ & \\
\hline \multicolumn{5}{|c|}{ Simple Matching (Sneath and Sokal 1973)... } \\
\hline BR 4 & $0,91 \pm 0,07$ & \multirow{2}{*}{\multicolumn{2}{|c|}{$097+007$}} & \multirow[b]{4}{*}{$0,86 \pm 0,08$} \\
\hline Embrapa 17 & $0,75 \pm 0,07$ & & & \\
\hline Embrapa 53 & $0,70 \pm 0,06$ & $0,63 \pm 0,06$ & $0,96 \pm 0,05$ & \\
\hline \multirow[t]{2}{*}{ BRS 148} & \multirow{2}{*}{\multicolumn{3}{|c|}{$\begin{array}{l}0,66 \pm 0,07 \quad 0,63 \pm 0,06 \quad 0,52 \pm 0,06 \\
\ldots \ldots \ldots \ldots . \text { Coeficiente de Yule } \ldots \ldots \ldots \ldots \ldots \ldots \ldots\end{array}$}} & \\
\hline & & & & \\
\hline BR 4 & \multicolumn{3}{|c|}{$0,97 \pm 0,07$} & \multirow[b]{4}{*}{$0,93 \pm 0,09$} \\
\hline Embrapa 17 & $0,70 \pm 0,23$ & \multicolumn{2}{|l|}{$0,98 \pm 0,06$} & \\
\hline Embrapa 53 & $0,54 \pm 0,16$ & $0,20 \pm 0,28$ & $0,99 \pm 0,02$ & \\
\hline BRS 148 & $0,38 \pm 0,30$ & $0,21 \pm 0,38$ & $-0,35 \pm 0,26$ & \\
\hline \multicolumn{5}{|c|}{ Coeficiente de Baroni-Urbani Buser ......... } \\
\hline BR 4 & \multicolumn{3}{|l|}{$0,92 \pm 0,06$} & \multirow[b]{4}{*}{$0,88 \pm 0,07$} \\
\hline Embrapa 17 & $0,78 \pm 0,06$ & \multicolumn{2}{|l|}{$0,93 \pm 0,06$} & \\
\hline Embrapa 53 & $0,73 \pm 0,05$ & $0,67 \pm 0,06$ & $0,96 \pm 0,04$ & \\
\hline BRS 148 & $0,69 \pm 0,06$ & $0,66 \pm 0,06$ & $0,57 \pm 0,06$ & \\
\hline \multicolumn{5}{|c|}{......Coeficiente de Sorensen-Dice. } \\
\hline BR 4 & \multicolumn{3}{|c|}{$0,94 \pm 0,05$} & \multirow[b]{4}{*}{$0,90 \pm 0,06$} \\
\hline Embrapa 17 & $0,82 \pm 0,05$ & \multicolumn{2}{|l|}{$0,94 \pm 0,05$} & \\
\hline Embrapa 53 & $0,78 \pm 0,05$ & $0,74 \pm 0,05$ & $0,97 \pm 0,03$ & \\
\hline BRS 148 & $0,75 \pm 0,05$ & $0,73 \pm 0,05$ & $0,66 \pm 0,06$ & \\
\hline
\end{tabular}

bandas, nas suas respectivas posições, considerando portanto cada tipo de banda como uma variável binária.

O coeficiente de Jaccard (Jaccard, 1908) é talvez o coeficiente binário mais amplamente utilizado para calcular medidas de similaridades entre eletroforegramas. Ele mede a proporção de bandas presentes nos dois perfis, ou seja, divide o número de bandas comuns pelo número total de bandas nos dois eletroforegramas. Outro coeficiente muito utilizado na análise de dados derivados de géis de eletroforese, derivado do coeficiente de Jaccard, foi desenvolvido por Dice (1945) e também utiliza a posição de banda, mas adiciona maior peso às bandas que coincidem. O coeficiente de Nei e Li (Nei \& Li, 1979) mede a probabilidade de um produto amplificado em uma amostra também ser amplificado em outra.

O coeficiente de Pearson é baseado na curva de valores de intensidade (densidade) ao longo do eletroforegrama. É considerado o coeficiente mais robusto e objetivo já que todo o perfil do eletroforegrama pode ser comparado e a identificação subjetiva das bandas pode ser omitida. Vale lembrar que a identificação automática das bandas, possibilitada pela maioria dos softwares de análise de géis, muitas vezes não ocorre com perfeição e necessita da intervenção do analista para evitar erros grosseiros. Isso pode ser um trabalho bastante enfadonho. Por isso, a grande vantagem do coeficiente de Pearson é que esse coeficiente possibilita comparar amostras sem a necessidade de identificar as bandas em cada eletroforegrama. Verifica-se, no entanto, que esta facilidade é mais adequada para comparações dentro do mesmo gel, ou quando se utilizam géis pré-confeccionados.

Embora tenha sido aplicado um procedimento cuidadoso de normalização dos eletroforegramas em diferentes géis convencionais, dificilmente se consegue bons resultados comparando os perfis, especialmente quando os perfis são muito similares, como é o caso das quatro cultivares estudadas neste trabalho (Figura 1).

A análise numérica de todos os eletroforegramas, baseada nos diferentes coeficientes de similaridade, revelou quatro grupos bem distintos, formados na maioria por apenas uma cultivar (dendrogramas não mostrados).

As médias e desvios padrões dos coeficientes de similaridade dentro da cultivar e entre cul- 
TABELA 2. Valores médios de similaridade comparando as amostras de semente única dentro da cultivar e entre cultivares de triticale, através de cinco coeficientes, considerando vários números de classes de intensidade das bandas.

\begin{tabular}{|c|c|c|c|c|c|c|}
\hline \multirow{3}{*}{ Cultivares } & \multicolumn{6}{|c|}{ Pearson product moment correlation coefficient } \\
\hline & binomial & 3 classes & 6 classes & 11 classes & 21 classes & 101 classes \\
\hline & \multicolumn{6}{|c|}{ Similaridade (média \pm DP) } \\
\hline $\mathrm{BR} 4(n=54)$ & $0,81 \pm 0,15$ & $0,76 \pm 0,12$ & $0,75 \pm 0,13$ & $0,73 \pm 0,14$ & $0,73 \pm 0,15$ & $0,72 \pm 0,16$ \\
\hline Embrapa $17(n=56)$ & $0,77 \pm 0,24$ & $0,72 \pm 0,14$ & $0,67 \pm 0,16$ & $0,65 \pm 0,18$ & $0,63 \pm 0,19$ & $0,62 \pm 0,19$ \\
\hline Embrapa $53(n=57)$ & $0,90 \pm 0,11$ & $0,78 \pm 0,14$ & $0,72 \pm 0,18$ & $0,70 \pm 0,20$ & $0,69 \pm 0,21$ & $0,68 \pm 0,21$ \\
\hline BRS $148(n=58)$ & $0,68 \pm 0,20$ & $0,66 \pm 0,15$ & $0,66 \pm 0,17$ & $0,66 \pm 0,17$ & $0,65 \pm 0,18$ & $0,64 \pm 0,19$ \\
\hline BR 4 x Emb 17 & $0,39 \pm 0,19$ & $0,29 \pm 0,18$ & $0,28 \pm 0,19$ & $0,28 \pm 0,20$ & $0,28 \pm 0,21$ & $0,28 \pm 0,21$ \\
\hline BR 4 x Emb 53 & $0,27 \pm 0,10$ & $0,35 \pm 0,12$ & $0,41 \pm 0,15$ & $0,42 \pm 0,17$ & $0,43 \pm 0,17$ & $0,43 \pm 0,18$ \\
\hline BR 4 x BRS 148 & $0,20 \pm 0,16$ & $0,19 \pm 0,17$ & $0,24 \pm 0,17$ & $0,24 \pm 0,17$ & $0,24 \pm 0,17$ & $0,24 \pm 0,18$ \\
\hline Emb $17 \times$ Emb 53 & $0,10 \pm 0,14$ & $0,15 \pm 0,17$ & $0,24 \pm 0,20$ & $0,27 \pm 0,22$ & $0,28 \pm 0,22$ & $0,29 \pm 0,22$ \\
\hline Bem 17 x BRS 148 & $0,11 \pm 0,18$ & $0,02 \pm 0,19$ & $0,06 \pm 0,19$ & $0,06 \pm 0,20$ & $0,06 \pm 0,20$ & $0,07 \pm 0,20$ \\
\hline Emb $53 \times$ BRS 148 & $-0,14 \pm 0,12$ & $-0,13 \pm 0,15$ & $-0,06 \pm 0,17$ & $-0,04 \pm 0,17$ & $-0,03 \pm 0,18$ & $-0,01 \pm 0,18$ \\
\hline
\end{tabular}

\begin{tabular}{|c|c|c|c|c|c|c|}
\hline \multirow{3}{*}{ Cultivares } & \multicolumn{6}{|c|}{ Spearmen rank order correlation coefficient } \\
\hline & binomial & 3 classes & 6 classes & 11 classes & 21 classes & 101 classes \\
\hline & \multicolumn{6}{|c|}{ Similaridade (média \pm DP) } \\
\hline $\mathrm{BR} 4(\mathrm{n}=54)$ & $0,73 \pm 0,20$ & $0,77 \pm 0,12$ & $0,78 \pm 0,11$ & $0,78 \pm 0,12$ & $0,78 \pm 0,12$ & $0,78 \pm 0,12$ \\
\hline Embrapa $17(\mathrm{n}=56)$ & $0,73 \pm 0,22$ & $0,73 \pm 0,13$ & $0,73 \pm 0,13$ & $0,73 \pm 0,14$ & $0,73 \pm 0,14$ & $0,73 \pm 0,14$ \\
\hline Embrapa $53(\mathrm{n}=57)$ & $0,92 \pm 0,09$ & $0,79 \pm 0,13$ & $0,79 \pm 0,14$ & $0,79 \pm 0,14$ & $0,79 \pm 0,14$ & $0,79 \pm 0,14$ \\
\hline BRS $148(\mathrm{n}=58)$ & $0,64 \pm 0,21$ & $0,67 \pm 0,15$ & $0,68 \pm 0,14$ & $0,67 \pm 0,14$ & $0,67 \pm 0,14$ & $0,67 \pm 0,14$ \\
\hline BR 4 x Emb 17 & $0,34 \pm 0,22$ & $0,32 \pm 0,17$ & $0,34 \pm 0,15$ & $0,35 \pm 0,15$ & $0,35 \pm 0,16$ & $0,35 \pm 0,16$ \\
\hline BR $4 \times$ Emb 53 & $0,15 \pm 0,11$ & $0,31 \pm 0,12$ & $0,35 \pm 0,12$ & $0,35 \pm 0,12$ & $0,34 \pm 0,13$ & $0,34 \pm 0,12$ \\
\hline BR 4 x BRS 148 & $0,15 \pm 0,19$ & $0,20 \pm 0,17$ & $0,27 \pm 0,15$ & $0,28 \pm 0,15$ & $0,29 \pm 0,15$ & $0,29 \pm 0,15$ \\
\hline Emb 17 x Emb 53 & $0,04 \pm 0,16$ & $0,13 \pm 0,17$ & $0,18 \pm 0,17$ & $0,19 \pm 0,18$ & $0,19 \pm 0,18$ & $0,19 \pm 0,18$ \\
\hline Bem 17 x BRS 148 & $0,07 \pm 0,20$ & $0,04 \pm 0,18$ & $0,09 \pm 0,17$ & $0,09 \pm 0,17$ & $0,10 \pm 0,17$ & $0,10 \pm 0,17$ \\
\hline Emb 53 x BRS 148 & $-0,02 \pm 0,17$ & $-0,13 \pm 0,14$ & $-0,11 \pm 0,14$ & $-0,11 \pm 0,14$ & $-0,11 \pm 0,14$ & $-0,11 \pm 0,14$ \\
\hline
\end{tabular}

\begin{tabular}{lcccccc}
\hline \multirow{2}{*}{ Cultivares } & \multicolumn{5}{c}{ Percent similarity coefficient } \\
\cline { 2 - 7 } & binomial & 3 classes & 6 classes & 11 classes & 21 classes & 101 classes \\
\cline { 2 - 7 } & & \multicolumn{5}{c}{ Similaridade (média \pm DP) } \\
\hline BR 4 (n = 54) & $93,45 \pm 5,25$ & $87,60 \pm 5,80$ & $78,23 \pm 7,11$ & $74,14 \pm 7,73$ & $72,40 \pm 8,15$ & $70,93 \pm 8,61$ \\
Embrapa 17 $(\mathrm{n}=56)$ & $93,52 \pm 5,59$ & $84,88 \pm 5,95$ & $75,38 \pm 8,37$ & $71,99 \pm 9,94$ & $70,27 \pm 10,48$ & $68,69 \pm 11,15$ \\
Embrapa 53 (n = 57) & $96,99 \pm 3,37$ & $88,51 \pm 6,39$ & $79,39 \pm 8,59$ & $75,75 \pm 9,25$ & $74,07 \pm 9,74$ & $72,69 \pm 10,17$ \\
BRS 148 (n= 58) & $89,35 \pm 6,73$ & $83,23 \pm 6,72$ & $73,62 \pm 8,48$ & $69,78 \pm 9,27$ & $68,15 \pm 9,85$ & $66,51 \pm 10,48$ \\
BR 4 x Emb 17 & $81,60 \pm 5,22$ & $72,58 \pm 6,42$ & $61,15 \pm 8,05$ & $57,12 \pm 8,86$ & $55,25 \pm 9,31$ & $53,58 \pm 9,78$ \\
BR 4 x Emb 53 & $78,28 \pm 4,84$ & $72,09 \pm 5,63$ & $62,29 \pm 6,72$ & $58,60 \pm 7,43$ & $56,96 \pm 7,84$ & $55,60 \pm 8,22$ \\
BR 4 x BRS 148 & $74,66 \pm 5,54$ & $66,41 \pm 5,66$ & $56,89 \pm 6,86$ & $52,52 \pm 7,80$ & $50,76 \pm 8,22$ & $48,97 \pm 8,71$ \\
Emb 17 x Emb 53 & $73,72 \pm 5,05$ & $66,31 \pm 6,13$ & $58,71 \pm 6,99$ & $55,92 \pm 7,48$ & $54,61 \pm 7,75$ & $53,42 \pm 8,00$ \\
Bem 17 x BRS 148 & $72,56 \pm 5,08$ & $63,34 \pm 5,62$ & $52,88 \pm 6,29$ & $49,03 \pm 7,07$ & $47,21 \pm 7,37$ & $45,48 \pm 7,78$ \\
Emb 53 x BRS 148 & $65,87 \pm 5,93$ & $57,72 \pm 5,39$ & $46,83 \pm 5,98$ & $43,01 \pm 6,64$ & $41,14 \pm 6,93$ & $39,52 \pm 7,21$ \\
\hline
\end{tabular}


...Continuação Tabela 2

\begin{tabular}{|c|c|c|c|c|c|c|}
\hline \multirow{3}{*}{ Cultivares } & \multicolumn{6}{|c|}{ Modified Morisita's coefficient } \\
\hline & binomial & 3 classes & 6 classes & 11 classes & 21 classes & 101 classes \\
\hline & \multicolumn{6}{|c|}{ Similaridade (média \pm DP) } \\
\hline BR $4(\mathrm{n}=54)$ & $0,93 \pm 0,05$ & $0,91 \pm 0,05$ & $0,87 \pm 0,07$ & $0,85 \pm 0,08$ & $0,83 \pm 0,09$ & $0,82 \pm 0,10$ \\
\hline Embrapa $17(\mathrm{n}=56)$ & $0,92 \pm 0,10$ & $0,89 \pm 0,05$ & $0,85 \pm 0,08$ & $0,82 \pm 0,11$ & $0,80 \pm 0,12$ & $0,78 \pm 0,13$ \\
\hline Embrapa $53(\mathrm{n}=57)$ & $0,97 \pm 0,03$ & $0,92 \pm 0,05$ & $0,87 \pm 0,08$ & $0,85 \pm 0,10$ & $0,84 \pm 0,11$ & $0,82 \pm 0,12$ \\
\hline BRS $148(\mathrm{n}=58)$ & $0,89 \pm 0,09$ & $0,87 \pm 0,06$ & $0,82 \pm 0,09$ & $0,80 \pm 0,11$ & $0,78 \pm 0,12$ & $0,77 \pm 0,13$ \\
\hline BR 4 x Emb 17 & $0,81 \pm 0,07$ & $0,74 \pm 0,07$ & $0,66 \pm 0,10$ & $0,62 \pm 0,11$ & $0,60 \pm 0,12$ & $0,59 \pm 0,13$ \\
\hline BR 4 x Emb 53 & $0,78 \pm 0,05$ & $0,76 \pm 0,05$ & $0,72 \pm 0,08$ & $0,69 \pm 0,09$ & $0,68 \pm 0,10$ & $0,67 \pm 0,11$ \\
\hline BR 4 x BRS 148 & $0,75 \pm 0,06$ & $0,69 \pm 0,07$ & $0,61 \pm 0,11$ & $0,57 \pm 0,12$ & $0,55 \pm 0,12$ & $0,53 \pm 0,13$ \\
\hline Emb 17 x Emb 53 & $0,74 \pm 0,05$ & $0,70 \pm 0,07$ & $0,66 \pm 0,09$ & $0,64 \pm 0,10$ & $0,63 \pm 0,11$ & $0,62 \pm 0,11$ \\
\hline Bem 17 x BRS 148 & $0,72 \pm 0,07$ & $0,64 \pm 0,06$ & $0,55 \pm 0,09$ & $0,51 \pm 0,10$ & $0,49 \pm 0,11$ & $0,47 \pm 0,11$ \\
\hline Emb 53 x BRS 148 & $0,66 \pm 0,07$ & $0,59 \pm 0,06$ & $0,49 \pm 0,08$ & $0,45 \pm 0,09$ & $0,43 \pm 0,10$ & $0,42 \pm 0,11$ \\
\hline \multirow{3}{*}{ Cultivares } & \multicolumn{6}{|c|}{ Gower general similarity coefficient } \\
\hline & binomial & 3 classes & 6 classes & 11 classes & 21 classes & 101 classes \\
\hline & \multicolumn{6}{|c|}{ Similaridade (média \pm DP) } \\
\hline $\mathrm{BR} 4(\mathrm{n}=54)$ & $0,92 \pm 0,06$ & $0,89 \pm 0,05$ & $0,87 \pm 0,05$ & $0,86 \pm 0,05$ & $0,86 \pm 0,05$ & $0,86 \pm 0,05$ \\
\hline Embrapa $17(\mathrm{n}=56)$ & $0,94 \pm 0,06$ & $0,85 \pm 0,06$ & $0,82 \pm 0,06$ & $0,82 \pm 0,06$ & $0,82 \pm 0,06$ & $0,82 \pm 0,06$ \\
\hline Embrapa $53(\mathrm{n}=57)$ & $0,96 \pm 0,05$ & $0,88 \pm 0,07$ & $0,85 \pm 0,07$ & $0,84 \pm 0,06$ & $0,84 \pm 0,06$ & $0,83 \pm 0,06$ \\
\hline BRS $148(\mathrm{n}=58)$ & $0,89 \pm 0,07$ & $0,85 \pm 0,06$ & $0,84 \pm 0,06$ & $0,84 \pm 0,06$ & $0,84 \pm 0,05$ & $0,84 \pm 0,05$ \\
\hline BR 4 x Emb 17 & $0,76 \pm 0,06$ & $0,75 \pm 0,07$ & $0,74 \pm 0,07$ & $0,75 \pm 0,06$ & $0,75 \pm 0,06$ & $0,76 \pm 0,06$ \\
\hline BR 4 x Emb 53 & $0,79 \pm 0,05$ & $0,75 \pm 0,06$ & $0,75 \pm 0,05$ & $0,75 \pm 0,05$ & $0,76 \pm 0,05$ & $0,76 \pm 0,05$ \\
\hline BR 4 x BRS 148 & $0,71 \pm 0,06$ & $0,71 \pm 0,05$ & $0,75 \pm 0,05$ & $0,75 \pm 0,04$ & $0,76 \pm 0,04$ & $0,77 \pm 0,04$ \\
\hline Emb 17 x Emb 53 & $0,72 \pm 0,05$ & $0,68 \pm 0,07$ & $0,70 \pm 0,07$ & $0,71 \pm 0,06$ & $0,72 \pm 0,06$ & $0,72 \pm 0,06$ \\
\hline Bem 17 x BRS 148 & $0,68 \pm 0,06$ & $0,67 \pm 0,07$ & $0,69 \pm 0,06$ & $0,70 \pm 0,05$ & $0,71 \pm 0,05$ & $0,72 \pm 0,06$ \\
\hline Emb 53 x BRS 148 & $0,58 \pm 0,06$ & $0,62 \pm 0,06$ & $0,65 \pm 0,06$ & $0,67 \pm 0,05$ & $0,67 \pm 0,05$ & $0,68 \pm 0,05$ \\
\hline
\end{tabular}

tivares, calculados por seis medidas diferentes, considerando apenas a presença ou ausência das bandas (Tabela 1), demonstram que apesar do baixo polimorfismo intervarietal, os coeficientes médios de similaridade dentro das cultivares sempre foram maiores do que entre cultivares, o que indica alto poder discriminatório dos testes. Os seis coeficientes de similaridade tiveram um comportamento muito similar entre as diferentes comparações intravarietais e entre cultivares, tendo uma variação também muito homogênea, medida pelo desvio padrão. Os coeficientes de similaridade intravarietal, utilizando as diferentes medidas baseadas em dados binários, foram sempre maiores que $0,82(82 \%)$, sendo que o maior índice de similaridade entre genótipos foi encontrado entre as cultivares Embrapa 17 e BR 4, e intravarietal na cultivar Embrapa 53 (Tabela 1). Isso indica que a cultivar Embrapa 53 produz eletroforegramas de gliadinas muito homogêneos entre as diferentes amostras de semente única. Ao contrário, a cultivar BRS 148 apresentou o menor índice intravarietal em todos os coeficientes, sendo portanto a cultivar que mostra maior variabilidade nos eletroforegramas em diferentes amostras. $\mathrm{O}$ alto índice de similaridade entre as cultivares Embrapa 17 e BR 4 indica uma estreita relação genotípica entre os dois genótipos.

De forma geral, os eletroforegramas de gliadinas das cultivares Embrapa 53 e BRS 148 apresentaram o menor índice de similaridade, em todos os coeficientes estudados, sendo portanto as duas cultivares mais distantes genotipicamente. A principal dedução desses resultados, entretanto, é que os diferentes coeficientes de similaridade estudados apresentam resultados altamente relacionados, sendo ambos boas medidas para a comparação de genótipos. Fica, portanto, difícil escolher qual seria o melhor coeficiente.

Em programas de identificação de cultivares, busca-se exaustivamente métodos que permitam uma alta discrimina- 


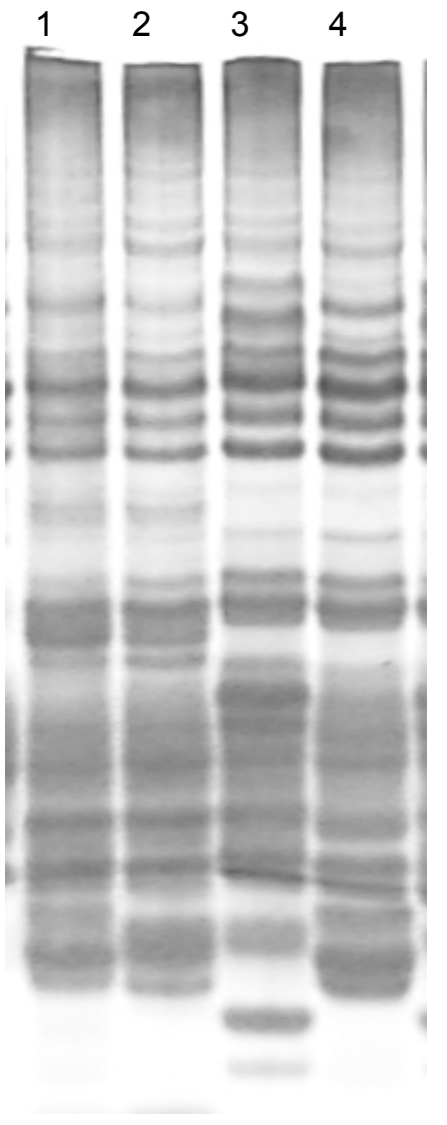

FIG. 1. Exemplo representativo dos eletroforegramas de gliadinas de quatro cultivares de triticale.

(1) BRS 148, (2) BR 4, (3) Embrapa 53, (4) Embrapa 17.

O topo da figura é a origem (pólo positivo).

ção entre as amostras de cultivares diferentes e baixa variação ou discriminação entre amostras da mesma cultivar. No entanto, o método não deve ser tão detalhista ao ponto de detectar muita variação entre amostras do mesmo genótipo, como é o caso do coeficiente de Yule. Este seria uma ótima medida, não fosse a elevada variação, evidenciada pelos desvios padrões (Tabela 1). Os demais coeficientes baseados em dados binários apresentam uma natureza muito similar quanto a esses parâmetros.

Na Tabela 2 encontram-se os valores médios e desvios padrões de similaridades, utilizando coeficientes que consideram a intensidade das bandas, em diferentes números de classes de intensidade. Pode-se observar que as medidas de similaridade levando em consideração as intensidades das bandas refletem a grande variabilidade do parâmetro intensidade das bandas e que a normalização entre géis não é suficiente para minimizar essa variação o suficiente. Observou-se, no entanto, que quando as comparações são feitas dentro do mesmo gel, a intensidade pode ter valor na discriminação entre genótipos (dados não mostrados).

Com relação à similaridade intra e intervarietal, esses coeficientes mostraram resultados equivalentes entre si e em relação aos coeficientes baseados em dados binários. No entanto, com o aumento no número de classes de intensidade esses coeficientes variam de forma diferenciada, havendo, entretanto, uma tendência geral de perda de confiabilidade nos resultados. Por exemplo, os coeficientes Percent Similarity e Modified Morista mostram um aumento na variabilidade dos resultados quando aumenta o número de classes de intensidade. O coeficiente de Pearson mostra claramente a perda de poder discriminatório com o aumento no número de classes de intensidade, isto é, diminui a similaridade intravarietal e aumenta a similaridade intervarietal. O coeficiente de Spearman se mostrou menos sensível ao aumento no número de classes de intensidade (Tabela 2).

Apesar do grande poder discriminatório entre cultivares, os coeficientes de Pearson e de Spearman apresentaram desvios padrões relativamente altos, o que evidencia uma alta variação entre valores relacionados. O coeficiente de Gower parece ser mais estável em termos de variação.

A Figura 2 mostra a distribuição de densidade média e o desvio padrão das gliadinas ao longo do perfil de bandas das quatro cultivares de triticale estudadas neste trabalho. Os gráficos evidenciam a alta variabilidade na densidade das bandas, sendo este, portanto, um parâmetro pouco confiável na análise de polimorfismos de gliadinas em triticale. Observase também a alta similaridade entre os perfis das diferentes cultivares, havendo maior concentração de proteínas nas regiões da banda 6 a 11 e da banda 18 a 20 . O processo de detecção automática das bandas pelo programa Diversity Database é bastante eficiente, mas algumas bandas parecem ter sido identificadas equivocadamente. Este problema pode ser corrigido por uma inspeção visual antes da geração dos resultados, mas prejudica o objetivo de automação do processo.

\section{CONCLUSÕES}

- O estudo demonstra a viabilidade de identificar cultivares de triticale pela comparação numérica dos dados de eletroforese de gliadinas, obtidos das amostras, com uma biblioteca de eletroforegramas. Esse procedimento garante uma comparação mais objetiva, em relação à análise visual dos eletroforegramas;

- a intensidade das bandas nos eletroforegramas de gliadinas é muito variável, sendo portanto um parâmetro pouco confiá- 
BR 4

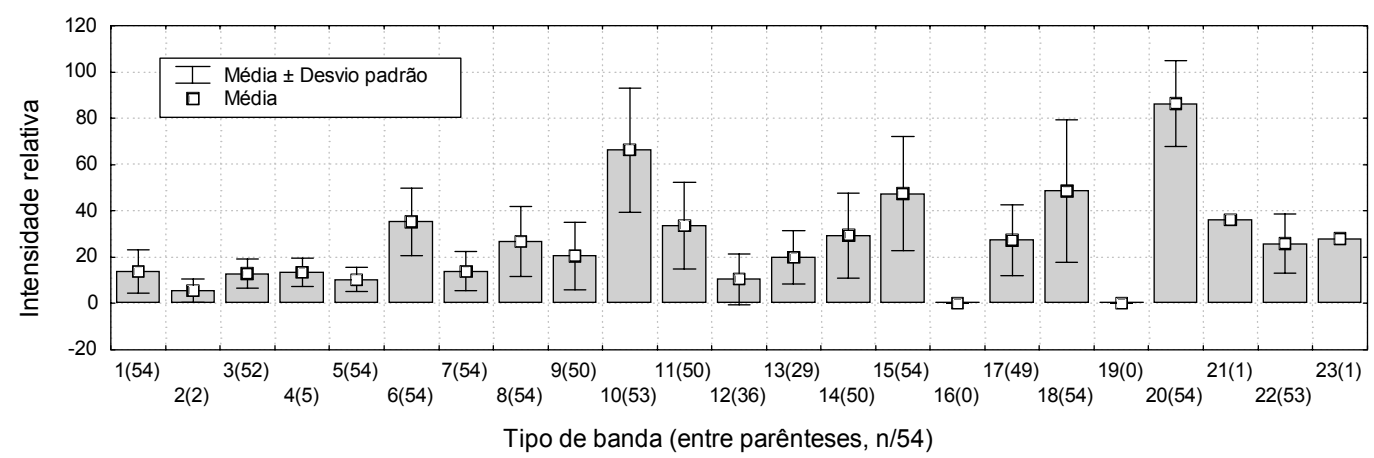

\section{Embrapa 53}

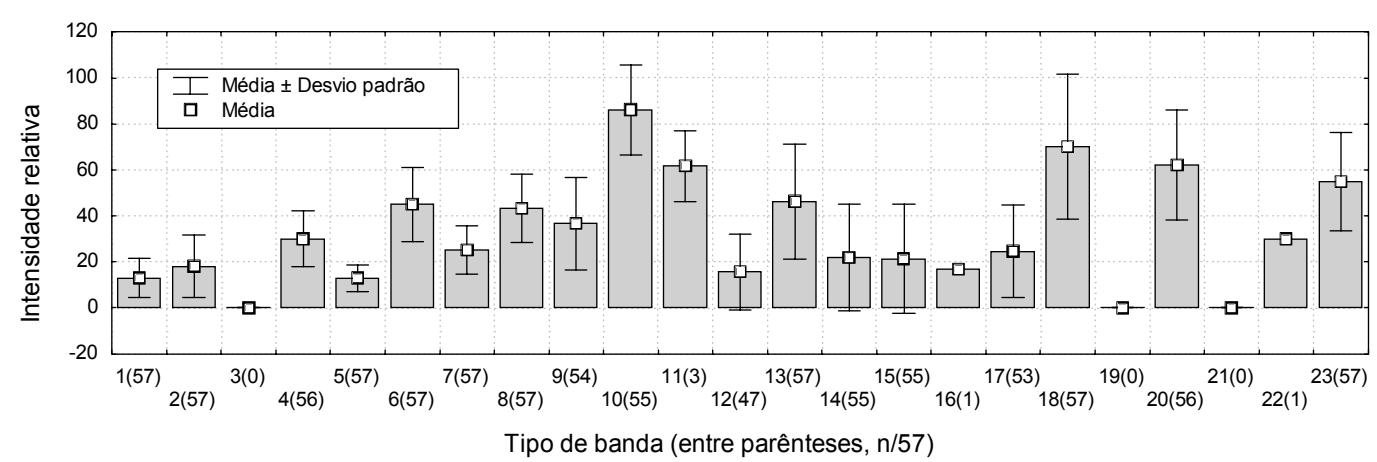

\section{Embrapa 17}

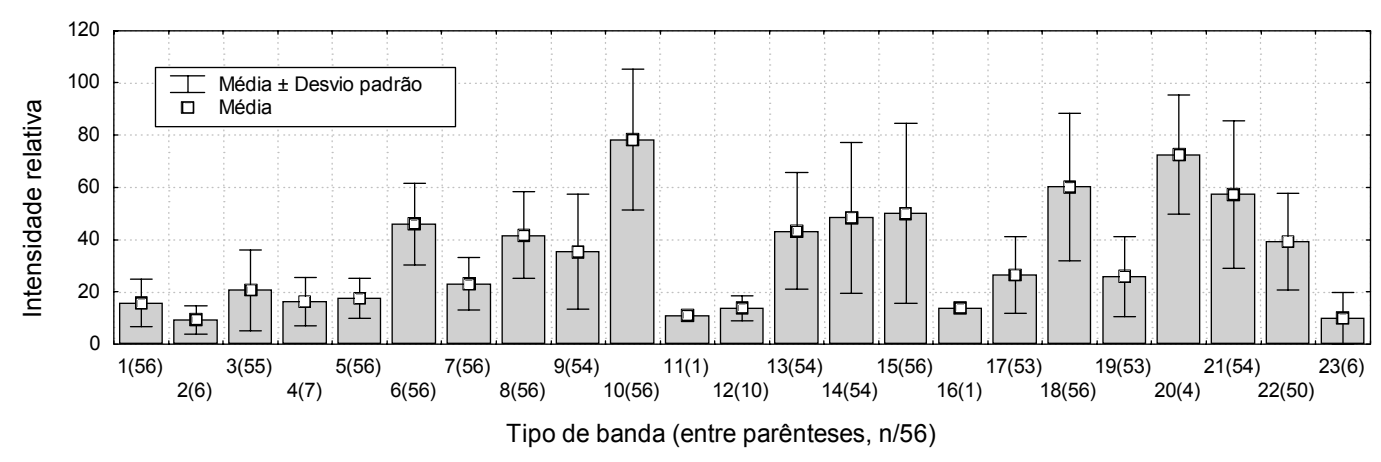

\section{BRS 148}

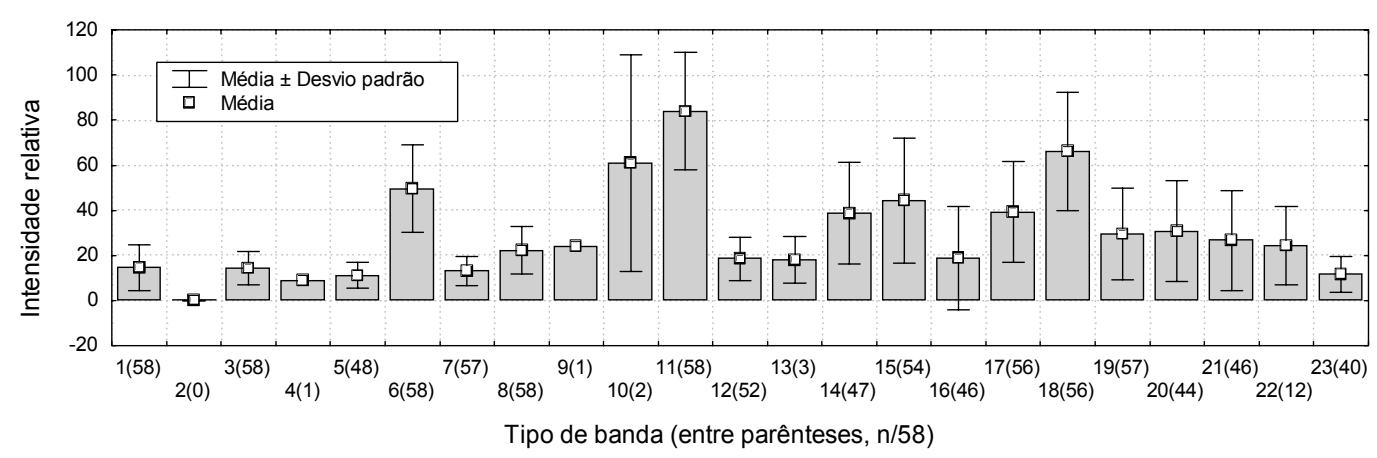

FIG. 2. Intensidade média, desvio padrão e freqüência dos diferentes tipos de bandas encontrados em quatro cultivares de triticale. 
vel na análise de polimorfismos de gliadinas em triticale. Ainda com relação ao parâmetro intensidade, há uma perda progressiva na confiabilidade dos resultados de comparação com o aumento no número de classes de intensidade.

\section{AGRADECIMENTOS}

Os autores agradecem à assistência técnica de Maria Alice Castro.

\section{REFERÊNCIAS}

BIETZ, J.A. \& SIMPSON, D.G. Electrophoresis and chromatography of wheat proteins: available methods, and procedures for statistical evaluation of the data. Journal of Chromatography, Amsterdam, v.624, n.8, p.53-80, 1992.

COOKE, R.J. The characterization and identification of crop cultivars by electrophoresis. Electrophoresis, Weinheim, v.5, n.1, p.5973, 1984.

COOKE, R.J. Gel electrophoresis for the identification of plant varieties. Journal of Chromatography, Amsterdam, v.698, n.1-2, p.281-299, 1995a.

DICE, L.R. Measures of the amount of ecological association between species. Journal of Ecology, New York, v.26, p.297302, 1945.

JACCARD, P. Nouvelles recherches sur la distribution florale. Bulletin de la Société Vaudense des Sciences Naturelles, v.44, p.223-270, 1908.

KOVACH, W.L. MVSP Plus, version 2.1. Wales: Kovach Computing Services. 1993.

MANLY, B.F.J. Multivariate statistical methods: a primer. London: Chapman \& Hall, 1994. 215p.

NEI, M. \& LI, W. Mathematical model for studying genetic variation in terms of restriction endonucleases. Proceedings of the National Academy of Sciences of the USA, Washington, v.76, p.5269-5273, 1979 .

SINGH, R.S.; JAIN, S.K. \& QUALSET, C.O. Protein electrophoresis as an aid to oat variety identification. Euphytica, Wageningen, v.22, n.1, p.98-105, 1973.

SNEATH, P.H.A. \& SOKAL, R.R. Numerical taxonomy. San Francisco: Freeman, 1973. 573p.

STATSOFT. STATISTICA for Windows [Computer program manual]. Tulsa: StatSoft, 1998.

SWOFFORD, D.L. \& OLSEN, G.J. Phylogeny reconstruction. In: HILLIS, D.M. \& MORITZ, C. (eds.). Molecular systematics. Sunderland: Sinauer Associates, 1990. p.411-501.

UPOV. International convention for the protection of new varieties of plants. Geneva, 1988. s.p. 